\title{
Experimental investigations on tetrahydrofuran - methane - water system: Rapid methane gas storage in hydrates
}

\author{
Burla Sai Kiran ${ }^{1}$, Kandadai Sowjanya ${ }^{1}$, Pinnelli S.R. Prasad ${ }^{1, *}$, and Ji-Ho Yoon ${ }^{2}$ \\ ${ }^{1}$ Gas Hydrate Division, CSIR - National Geophysical Research Institute (CSIR-NGRI), Hyderabad 500007, Telangana, India \\ ${ }^{2}$ Department of Energy and Resources Engineering and Department of Convergence Study on the Ocean Science and Technology, \\ Ocean Science and Technology (OST) School, Korea Maritime and Ocean University, Busan 606-791, Korea
}

Received: 10 September 2018 / Accepted: 20 November 2018

\begin{abstract}
This study reports methane $\left(\mathrm{CH}_{4}\right)$ gas storage capacity along with TetraHydroFuran (THF) as guest molecules in mixed hydrates. This process has been studied in two reactors of 100 and $400 \mathrm{~mL}$ capacity, having 4.5 and $7.5 \mathrm{~cm}$ internal diameter respectively, in non-stirred configuration. Experiments were conducted in each reactor at constant initial gas pressure $(7.5 \mathrm{MPa})$ and by increasing the height of the solution from 1 to $8 \mathrm{~cm}$, resulting in volume scale-up factor of 5 . The total $\mathrm{CH}_{4}$ gas uptake (moles) passes through a maximum at around $50 \%$ volume of the reactor indicating a transition from gas-rich to solution rich conditions. Observed variations in gas uptake are within $\pm 20 \%$ of the maximum, upon different solution volume from $35 \%$ to $70 \%$ of reactor's volume. Another set of experiments were conducted keeping the amount of the solution constant and increasing gas pressure in the range of 0.5-11.0 MPa. The gas uptake increased upon an increase in the gas pressure, but this is at least $40 \%$ less compared to the theoretical estimate. The stirring of solution or addition of promoter (Sodium Dodecyl Sulfate, SDS) is also not effective in increasing the gas consumption. Kinetics of gas uptake, in both stirred and non-stirred conditions, are quicker and $90 \%$ of gas consumption occurs in an hour after the hydrate nucleation event.
\end{abstract}

\section{Introduction}

Clathrate hydrates are the ice-like crystalline solids, often found in low temperature and high-pressure regions, namely in the certain arctic zone and oceanic sediments. The water molecules transform into porous ice in the presence of smaller hydrocarbons such as methane, ethane, propane, etc., or carbon dioxide; or hydrogen sulphide; and vacant pore spaces are occupied by these guest molecules. At the molecular level, a network of water molecules forms various polyhedral cages through hydrogen bonding, and the guest molecules are encased in cages. There are five types of polyhedral cages, commonly found in hydrates, in increasing size: pentagonal dodecahedron $\left(5^{12}\right.$-cage $)$, dodecahedron $\left(4^{3} 5^{6} 6^{3}\right.$ cage), tetrakaidecahedron $\left(5^{12} 6^{2}\right.$-cage), hexakaidecahedron $\left(5^{12} 6^{4}\right.$-cage $)$, and icosahedron $\left(5^{12} 6^{8}\right.$-cage $)$. Three common unit cells (sI, sII, and $\mathrm{sH}$ ) of gas hydrates are known to form from a few types of cages depending largely on the size and physical properties of the guest species. For example, the SI unit lattice consists of two $5^{12}$-cages and six $5^{12} 6^{2}$-cages, and the sII unit lattice has sixteen $5^{12}$-cages and eight $5^{12} 6^{4}$-cages. The sH hydrate comprises three different cages: three $5^{12}$-cages, two $4^{3} 5^{6} 6^{3}$-cages, and one $5^{12} 6^{8}$-cage [1].

\footnotetext{
* Corresponding author: psrprasad@ngri.res.in
}

The research on gas hydrates in earlier days remained purely as a scientific curiosity. Of late it has become a topic of great importance because of multi-facet applications in the energy sector, e.g., natural gas hydrates could be an energy source, large storage capacity could be utilised for gas storage and transportation, of course, depending on structural stability issues. Also, the property is much useful to sequester the greenhouse gas such as carbon-dioxide [2-7].

In particular, storage of Natural Gas (NG), where the dominant constituent is methane, is practised by different methods, such as Liquefaction (LNG) or Compressed $(\mathrm{CNG})$ or Adsorption (ANG) on suitable adsorbents or via clathrate hydrates (Solidified Natural Gas, SNG). LNG has a volumetric capacity of about $600 \mathrm{v} / \mathrm{v}$ and is a very active mode to transport NG from source to market, but not suitable for storage due to stringent temperature requirements and the continuous gas boiloff issues [8]. CNG $(\sim 200 \mathrm{v} / \mathrm{v}$ at $20 \mathrm{MPa})$ as a mode of storage requires high pressure for both production and storage and its inherent flammable/explosive nature, practically makes it hazardous for NG storage. In contrast, ANG requires adsorbing NG onto sorbents, like, activated carbon, Carbon Nanotubes (CNTs), graphene, Metal-Organic Frameworks (MOFs), etc. [9-12]. Given the history of the development of these materials for NG storage and known scale-up 
issues, there is a need to demonstrate process development. Furthermore, some of them are highly sensitive to moisture [13]. On the other hand, solid hydrates of natural gas (SNG) are stable at moderately high pressure $(5 \mathrm{MPa})$ and $279 \mathrm{~K}$. Under the assumption of complete cage occupancy ( 8 in sI and 16 small in sII) of methane gas, one unit of SNG upon dissociation can release 182 and 109 units of methane gas at $293 \mathrm{~K}$ and $0.1 \mathrm{MPa}$ from sI and sII structures. Thereby, the storage of gas in the form of SNG is a handy solution. The larger cages $\left(5^{12} 6^{4}\right)$ of sII in mixed hydrates are often occupied by the large co-guest molecules such as TetraHydroFuran (THF), resulting in significant reduction in the methane gas volumetric storage capacity. However, the thermodynamic stability conditions are vastly different than methane hydrates in sI [14].

However, noticeable drawbacks are in the form of the sluggish formation kinetics and inefficient water to hydrate conversion. The hydrates largely form at the gas-water interface and thus increase the surface area and helps in attaining higher hydrate conversion. The common methods for increasing clathrate formation kinetics, for example, use of high pressures (driving force), vigorous mechanical mixing, surfactants, or micron-sized ground/sieved ice particles, can be adopted in the laboratory environment [2, 15-17]. Also, the hydrates formed in a porous medium have shown rapid and efficient gas storage [18-22]. But the process of mixing of reactants continuously may be less cost-effective and impractical in real gas storage applications.

In the current study, we use THF, a well-known sII hydrate former, in stirred and unstirred configurations and investigate the formation of mixed methane/THF hydrates by systematically varying aq-THF amount keeping initial gas pressure at 7.5 $\mathrm{MPa}$ and also varying the initial methane gas pressure in the range $0.5-11.0 \mathrm{MPa}$ by keeping aq-THF solution constant. We used THF in stoichiometry (5.66 mol\%) for sII for the following reasons: it is well-known that under these conditions the hydrate system is known to have the highest thermodynamic stability $[16,23-25]$ and also the thermal stability at $0.1 \mathrm{MPa}$ is very high [14]. However, recently some studies using a lesser amount of THF indicated relatively higher methane gas uptake $[14,26,27]$ and also at much lower THF (1.0 mol \%) methane gas consumption is negligible, particularly in the absence of constant stirring [26]. However, experiments conducted with continuous stirring have shown significant gas consumption due to the phase change even with lower THF concentrations $[14,16]$. Furthermore, the addition of surfactants like sodium alkyl sulfates (C8-C16) along with low mol\% THF, helps in faster hydrate nucleation and higher gas uptake [28, 29]. Additionally detailed structural studies indicated that the hydrates formed with lesser amounts of THF are crystallised into mixtures of both sI and sII, while the hydrates with stoichiometry crystallise into sII [30, 31].

Hydrate phase equilibrium data for the methane + THF + water system [32-35] along with kinetic studies on bubbling reactor [36] and stirred tank reactor [14, 37] are reported in the literature. However, investigations of this system in quiescent unstirred setup under experimented conditions are at seldom in the literature [38, 39].
Veluswamy et al. [38, 39] have conducted studies on storage capacity and gas uptake kinetics in $\mathrm{CH}_{4}+\mathrm{THF}+\mathrm{H}_{2} \mathrm{O}$ system and have reported process scale-up using three different reactors, namely, in small (dia: $1.3 \mathrm{~cm})$, medium (dia: $5.1 \mathrm{~cm}$ ) and large (dia: $10.2 \mathrm{~cm}$ ) using 2, 53 and $220 \mathrm{~mL}$ of aq-THF solution at comparable methane gas pressure. However, such scale-up is deceptive as both diameter and volume (height) of the solution vary. Our motivation is to investigate the hydrate formation process with different amounts of aq-THF solution and initial gas pressure to understand the role of surface area, the volume of solution (height) and driving force (at a fixed volume of solution) as these are a relevant parameter in the gas storage process.

\section{Experimental method}

Figure 1 shows the schematic experimental layout employed in our study. We used two different SS-316 cylindrical reactor vessels of volume 100 and $400 \mathrm{~mL}$ for these experiments. Cold fluid (water + glycol mixture) was circulated in the vessel with the help of AN CYROLTCCB-40 circulator, to maintain the temperature inside it at the desired level. A platinum resistance thermometer (Pt100) inserted into the vessel was used to measure temperatures within $\pm 0.2 \mathrm{~K}$. The thermometer is placed in a thermowell, which was fixed closer to the bottom surface of the reactor vessel and therefore the measured temperature is always in liquid/hydrate phase. The pressure in the vessel was measured with a WIKA pressure transducer (WIKA, type A-10 for pressure range $0-16 \mathrm{MPa}$ ) and this sensor measures the variations in the vapour phase. The stoichiometric $5.66 \mathrm{~mol} \%$ THF was taken to prepare the aqueous solution. The solution filled to different heights in the vessel. Note that the vessel was flushed with methane gas to remove atmospheric gases. The required amount of methane gas was filled into the experimental vessel at ambient temperature $(\sim 298 \mathrm{~K})$ and the hydrate formation and dissociation cycles were conducted in standard isochoric configuration. The reactor vessel temperature was rapidly decreased by circulating the cold fluid. Since a high subcooling and driving force played a major role in hydrate crystallisation. The $P-T$ (pressure-temperature) profile was recorded at fixed time intervals. A sudden pressure drop was observed due to gas consumption in the hydrate cages. Within 1-2 h $90 \%$ of the total consumption happened. Then the reactor vessel was warmed up to $315 \mathrm{~K}$ to ensure complete hydrate dissociation and recovery of consumed methane gas. The complete process of hydrate formation and dissociation is shown in Figure 3. The process was repeated 2-3 times to ensure the reproducibility and the reusability of aqueous THF solution. The content of methane gas (moles) in the hydrate phase during the experiment at time $t$, is defined by the following equation:

$$
\Delta n \mathrm{H}, t=n \mathrm{~g}, 0-n \mathrm{~g}, t=P_{0} V / Z_{0} R T_{0}-\left(P_{t} V / Z_{t} R T_{t}\right),
$$

where, $Z$ is the compressibility factor calculated by the Peng-Robinson equation of state. The gas volume $(V)$ 


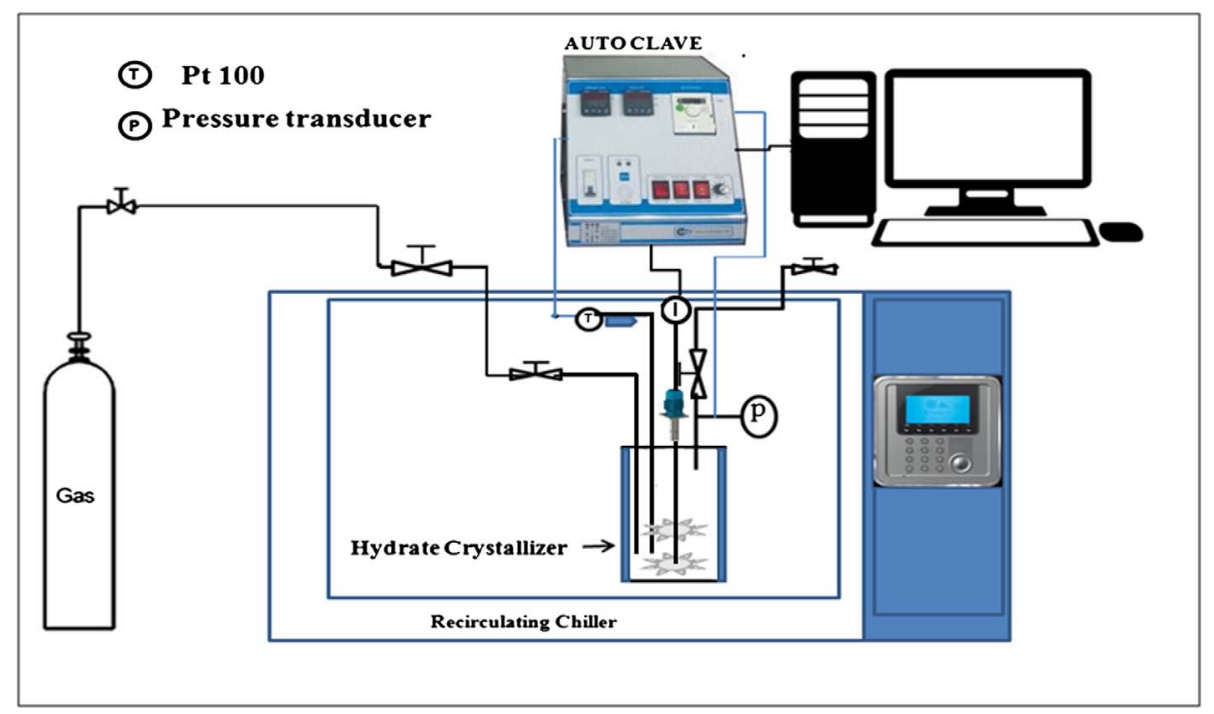

Fig. 1. Schematic diagram of the experimental set up.
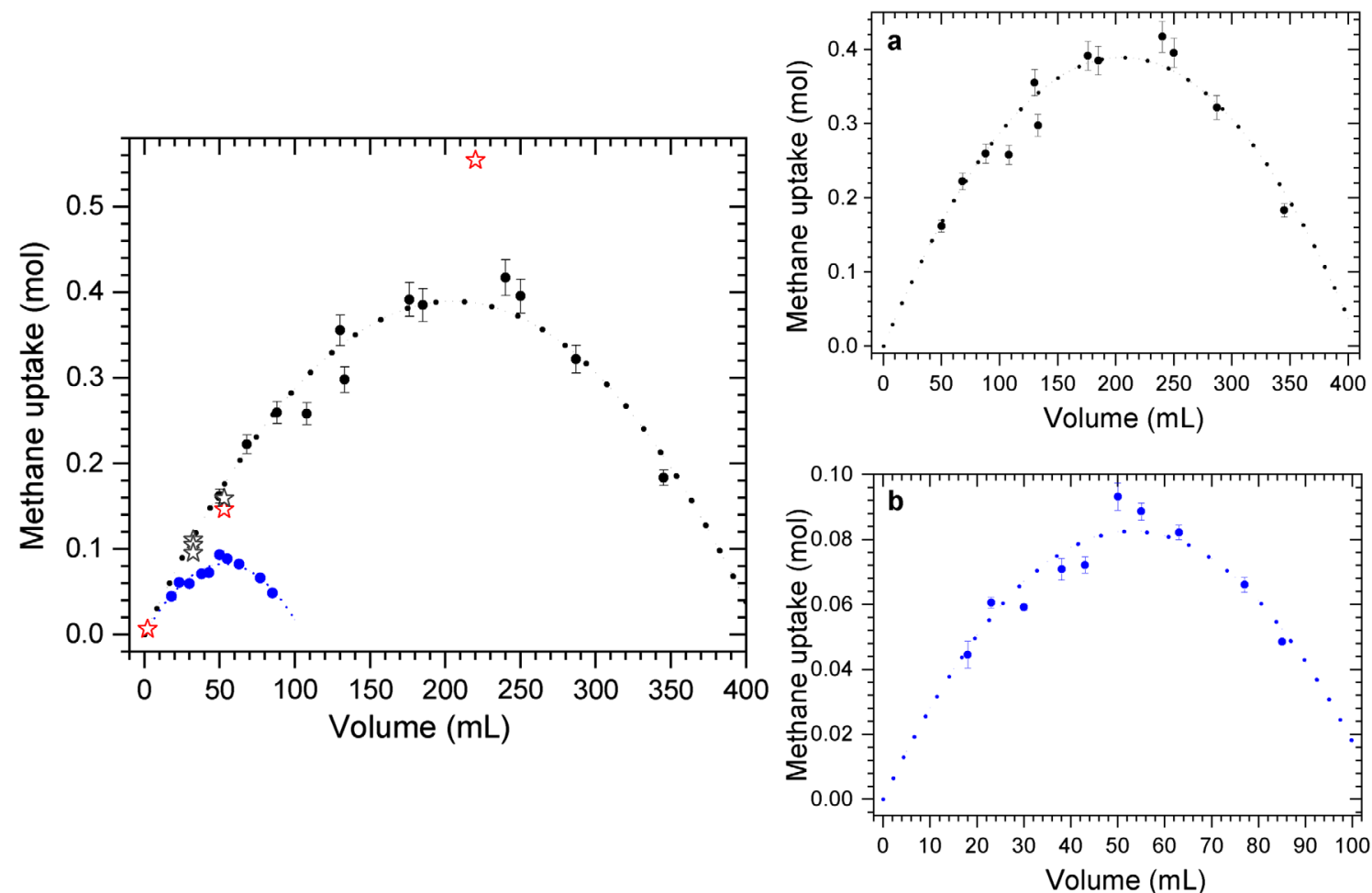

Fig. 2. Observed methane gas uptake in two reactors with by varying aq-THF volume (reactor-1 -

; reactor-2

. The symbols (放, 论) are the data points from Refs. [38, 39] and dotted lines are polynomial regression for the experimental points. For clarity experimental data for a) reactor-2 and b) reactor-1 are shown in expanded scale.

was assumed as constant during the experiments, i.e., the volume changes due to phase transitions were neglected. $n \mathrm{~g}, 0$ and $n \mathrm{~g}, t$ represent the number of moles of feed (methane) gas at hydrate onset point (zero time) and in the gas phase at any other time $t$, respectively.

\section{Results and discussion}

The decomposition temperature for pure THF hydrates (i.e., with empty $5^{12}$ cages) is $278 \mathrm{~K}$ at ambient pressure. However, the highest decomposition temperature for THF 
hydrates with $\mathrm{CH}_{4}$ molecules in $5^{12}$ cages is reported as $377 \mathrm{~K}$ at $1.5 \mathrm{GPa}$ pressure [40]. Therefore the mixed hydrates system with THF is interesting for methane gas storage and transportation applications. Moreover, thermodynamic stability is most significant for the $\mathrm{THF}+\mathrm{CH}_{4}$ system. Thus, the mixed hydrate system with THF has been chosen for assessing methane gas uptake capacity and kinetics using relatively larger amounts of the solution in two laboratory scale reactors. Generally, experiments on hydrates are conducted under stirring of the liquid phase which helps in improving the gas-liquid interfacial area. However, this could add cost while adopting this technology for large-scale gas storage systems.

We conducted first set of experiments in two reactor vessels with $4.5 \mathrm{~cm}$ (reactor-1) and $7.5 \mathrm{~cm}$ (reactor-2) internal diameters in non-stirred configuration. The volume of aqueous THF solution required to fill these reactors up to $1 \mathrm{~cm}$ height is 18 and $50 \mathrm{~mL}$ respectively. Identical initial methane gas pressure $(\sim 7.5 \mathrm{MPa})$ has been chosen in these experiments to probe the effect of surface area (internal diameter) and amount of aq-THF solution in the reactor. All the experiments were conducted in rapid mode, i.e., circulating bath was set to lower temperatures during hydrate formation and it was set at a high temperature to ensure rapid melting of hydrates. Typically entire process has been completed in $4-5 \mathrm{~h}$ duration. The trajectories of gas content in the vapour phase (pressure) and temperature variations over time are shown in supporting information (Fig. SI-1). Completion of the experimental cycle in reactor-2 is slower and this could be due to its size and less efficient heat transport. However, the process of gas consumption and release, respectively due to hydrate formation and dissociation in these reactors occurred in comparable time scales.

To get a better insight into the process, we systematically increased the height of the solution in two reactors. Figure 2 shows observed total gas uptake values with aq-THF volume. The process of hydrate formation was investigated in these reactors at different aq-THF volumes. Amount of aq-THF solution used in reactor-1 for heights of $1 \mathrm{~cm}$ and $6 \mathrm{~cm}$ respectively is 18 and $85 \mathrm{~mL}$. Similarly, in reactor- 2 we used 50 and $345 \mathrm{~mL}$ of aq-THF solution to attain the height of $1 \mathrm{~cm}$ and $8.5 \mathrm{~cm}$ respectively. Thus, the volume of aq-THF was increased by $4-7$ times by increasing the solution height. As shown in Figure 2 the methane gas uptake initially increased with an increase in the volume of the solution. At a later stage, the gas uptake decreased significantly at higher volume. Ke and Svartaas have shown that the molar water-gas ratio plays a vital role in hydrate formation and the gas uptake attains a maximum at some optimum ratio [41]. It is well known that the hydrates nucleate at the interface between the gas and water phases and it does not grow homogeneously as a planar front, but advances in the form of lobes that invade the water phase [42]. Volume expansion during hydrate growth causes water to flow out of the meniscus. The displaced water forms a coating on the surface which converts into thin hydrate layers. Rapid hydrate growth consumes the excess gas that dissolved by diffusive transport [42]. With increasing volume the diffusive transport increases to a certain height and the gas consumption is maximum at these heights, indicating an optimum level of solution in a reactor. The gas uptake gradually decreases with further height increase. The maximum gas uptake is 0.093 and $0.417 \mathrm{~mol}$ in these reactors is observed with 50 and $240 \mathrm{~mL}$ of aq-THF solution, which is about $50 \%$ of reactor capacity. Therefore, it is advantageous to fill the reactors to half of its volumetric capacity to achieve higher methane gas uptake in hydrate conversion. Initially, the amount of gas available for hydrate conversion is more as the vessels are not filled with excess solution and this region can be termed as "excess gas" region.

On the other hand "excess solution" in the vessel will lead to less space for gas molecules and therefore the lesser amount of gas will be utilised in the hydrate conversion process. It would be interesting to study the gas uptake process with the optimised solution under isobaric conditions. However, it is not possible with available experimental facilities in our laboratory.

It is worthwhile to compare the present results with recent studies of Veluswamy et al. [38]. They conducted experiments using fixed amounts of aq-THF solution $(2,53$ and $220 \mathrm{~mL})$ in three reactors (small - dia $1.3 \mathrm{~cm}$, medium - dia $5.1 \mathrm{~cm}$ and large - dia $10.2 \mathrm{~cm}$ ). The height of aq-THF solution in their medium and large reactors is $2.7 \mathrm{~cm}$. As shown in Figure 2, methane gas uptake values measured by Veluswamy et al. [38] in three different size reactors are in good agreement with our studies. However, all their experiments were conducted in "excess gas" region. The present studies vindicate that both the solution height and internal diameter of the reactor are important in maximising the gas uptake in hydrate formation.

Mixed hydrates with $\mathrm{CH}_{4}$ and THF as guest molecules forms sII structure with composition $16\left(\mathrm{CH}_{4}\right) \cdot 8(\mathrm{THF})$. $136 \mathrm{H}_{2} \mathrm{O}$ and methane and THF are encased in $5^{12}$ and $5^{12} 6^{4}$ cages. Thus the maximum methane gas uptake due to complete hydrate conversion will be $0.117 \mathrm{~mol} / \mathrm{mol}$. From Figure 2 it is seen that the maximum gas consumption of 0.093 and $0.417 \mathrm{~mol}$ occur at 50 and $240 \mathrm{~mL}$ of aq-THF solution and this accounts for only $30 \%$ of water usage in hydrate conversion. In other words, a large portion of aq-THF solution remained unutilized. Veluswamy et al., have reported a similar amount of hydrate conversions [38]. By the visual observations, they reported the formation of a craton like structure, concealing below part of the unconverted solution, and therefore became inaccessible for interacting with the gas phase. It is worthwhile to conduct experiments to enhance the accessibility of unconverted aq-THF solution to improve the hydrate conversion. Some of the classical ways are conducting the experiments with (i) higher gas pressures (driving force), (ii) in stirred conditions, and (iii) in the presence of additives like SDS. In the following paragraphs, we describe the experimental results under those conditions.

\section{Methane uptake at higher pressure}

Experiments were conducted in reactor- 2 with $185 \mathrm{~mL}$ of aq-THF solution at different initial methane gas pressures 


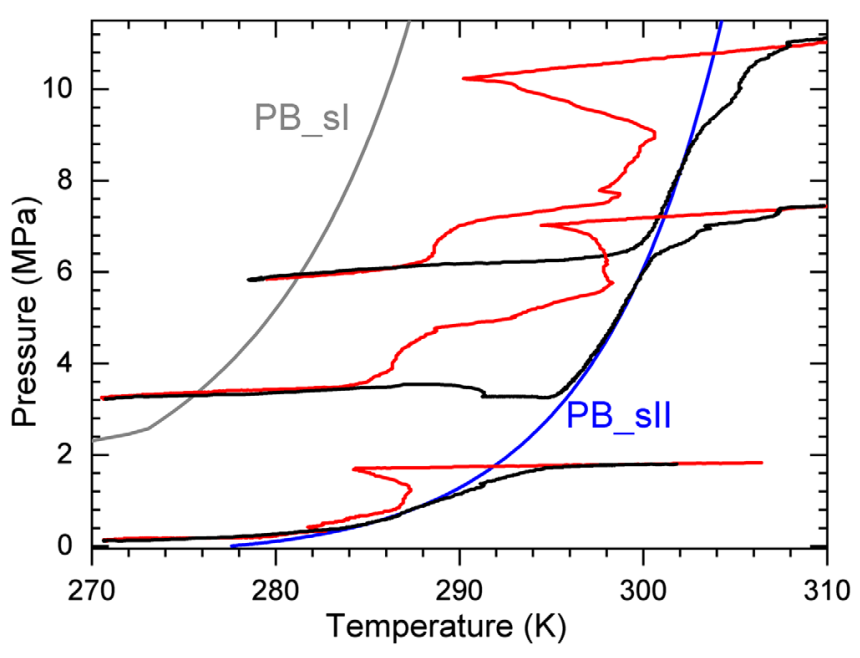

Fig. 3. $P-T$ trajectories of $\mathrm{THF}+\mathrm{CH}_{4}$ system at three different pressures along with the computed phase boundary curves (grey colour) for sI (CSMGem - pure $\mathrm{CH}_{4}$ ) and sII (blue colour; $\mathrm{CH}_{4}$ - THF). The red ( $)$ and the black lines $(-)$ are observed behaviour during cooling and warming cycles.

in the range $0.5-11.0 \mathrm{MPa}$. Figure 3, shows the pressuretemperature $(P-T)$ trajectory at three different pressures. The phase boundary curves for sI methane hydrates (calculated by CSMGem) and sII phase boundary curve for the $\mathrm{THF}+\mathrm{CH}_{4}+\mathrm{H}_{2} \mathrm{O}$ system [43] are also plotted. Observed $P-T$ trajectories vindicate that the experimental conditions can only promote sII hydrates and observed dissociation points are shifted to the right of the sII phase boundary curve. This could be due to faster warming employed in these experiments.

Figure 4 depicts the consumption of methane gas in hydrates at different pressures. We conducted the experiments in reactor- 2 with $185 \mathrm{~mL}$ of an aq-THF solution by varying the pressure in the range $0.5-10.3 \mathrm{MPa}$. As already stated the absolute amount of gas consumption is larger at this volume. Further, we also conducted another set of experiments with a lesser amount $(60 \mathrm{~mL})$ of aq-THF to understand the role of aq-THF volume. In all these experiments the gas consumption increased (grey colour lines) with gas pressure increase. Yoon [43] reported a gradual increase in the cage $\left(5^{12}\right)$ occupancy of $\mathrm{CH}_{4}$ molecules in $\mathrm{THF}-\mathrm{CH}_{4}$ with increasing pressure. The black line in Figure 4 represents the gas consumptions based on Yoon's cage occupancy at different pressures. It is worthwhile to note that the observed gas consumption is lower at least by $40-45 \%$ at higher pressures and it is much higher at low-pressure conditions. An increase in the gas consumption with a lesser amount of aq-THF is insignificant. Veluswamy et al., have conducted similar experiments at three pressures with $32.5 \mathrm{~mL}$ aq-THF solution and their results also compared in Figure 4. The observed gas consumption in all these cases shows an increasing trend with pressure increase, but it is significantly less compared to the predicted values. Thus, it is not feasible to populate all the small cages $\left(5^{12}\right)$ with methane molecules even at higher pressures. In other words, a significant amount of aq-THF

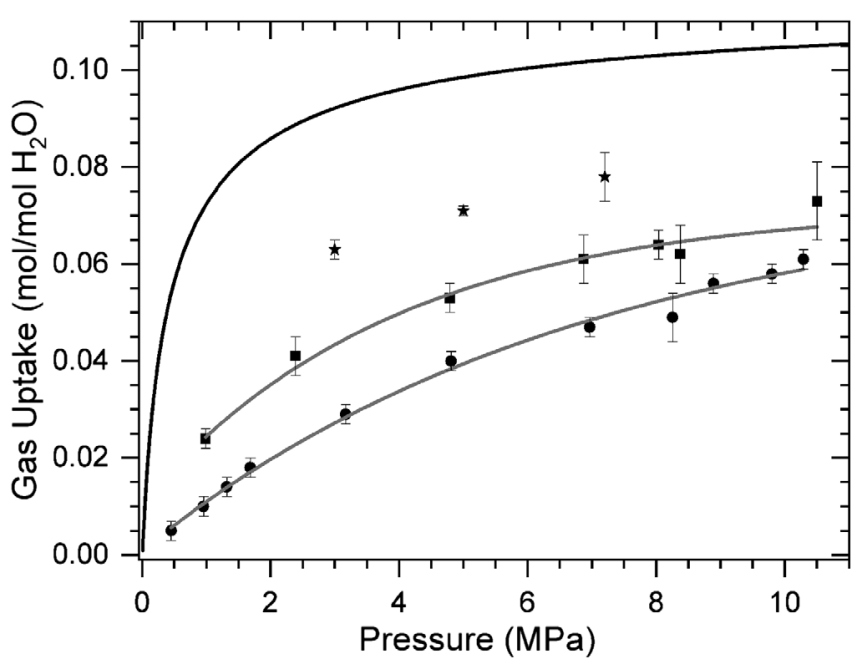

Fig. 4. A plot showing methane gas uptake $\left(\mathrm{mol} / \mathrm{mol}-\mathrm{H}_{2} \mathrm{O}\right)$ in hydrates at various initial gas pressures. The solid line is the computed gas uptake with small cage $\left(5^{12}\right)$ occupancy reported by Yoon [43]. Symbols $(\mathbf{\square})$ and $(\mathbf{0})$ represent gas uptake values with 60 and $185 \mathrm{~mL}$ of aq-THF solution in the reactor. $(\star)$ symbols are used to depict the similar observations with lesser (32.5 mL) amount of solution for hydrate formation (Ref. [39]). The dotted grey lines are guides to the eye.

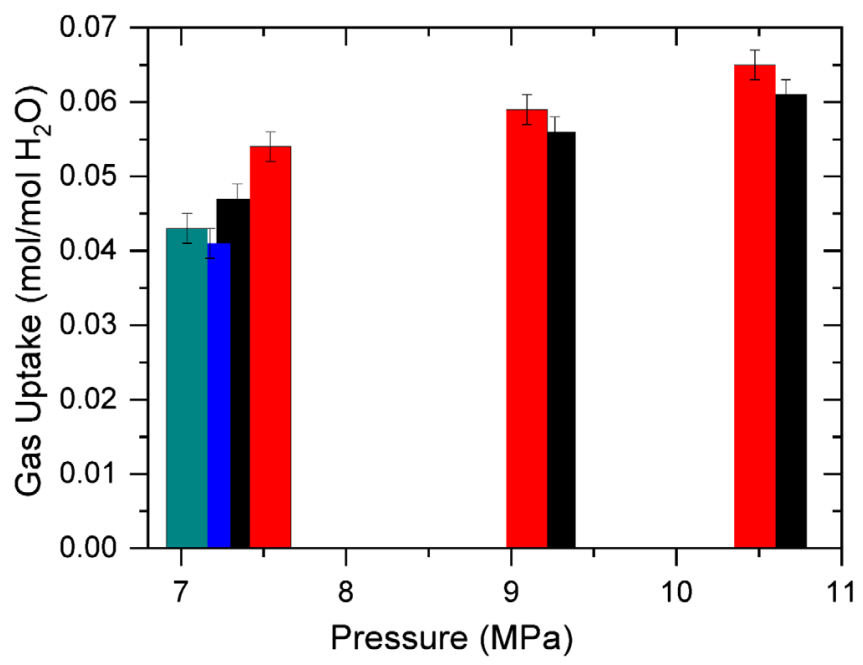

Fig. 5. Comparison of methane gas uptake in stirred (red bars) and non-stirred (black bars) conditions at different pressures. The green and blue bars represent the gas uptake with $0.5 \mathrm{wt} \%$ and $0.05 \mathrm{wt} \% \mathrm{SDS}$ in $\mathrm{THF}+\mathrm{CH}_{4}$ system.

solution remained inaccessible for hydrate conversion. One way of achieving a higher solution to hydrate conversion is to conduct the experiments in stirred reactors.

Figure 5 shows a comparison of gas uptake in stirred (red bars) and non-stirred (black bars) conditions at different pressures. It is seen the gas uptake is marginally $(\sim 5 \%)$ higher in stirred conditions, but still significantly less compared to the predicted value. We also investigated the hydrate formation in the $\mathrm{THF}+\mathrm{CH}_{4}$ system by adding 0.05 and $0.5 \mathrm{wt} \%$ SDS to an aq-THF solution. As shown 


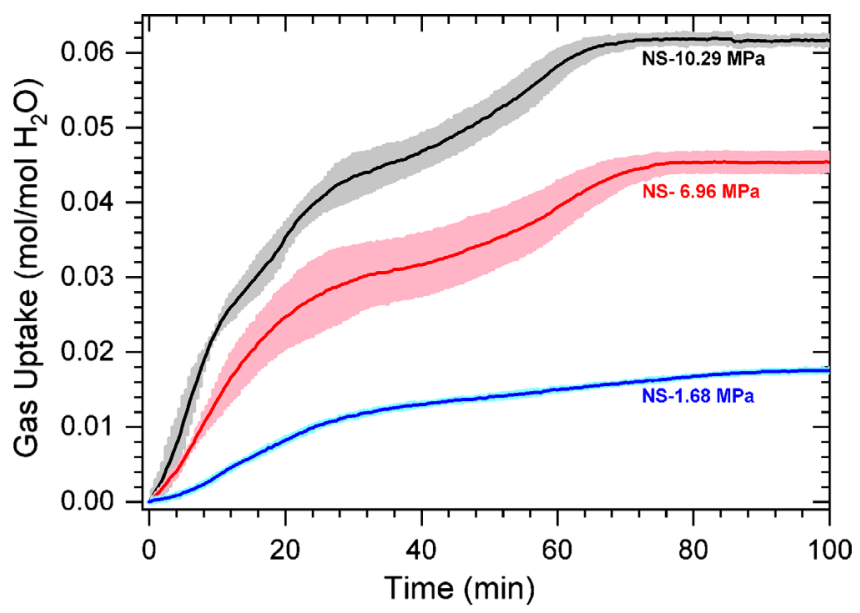

(a)

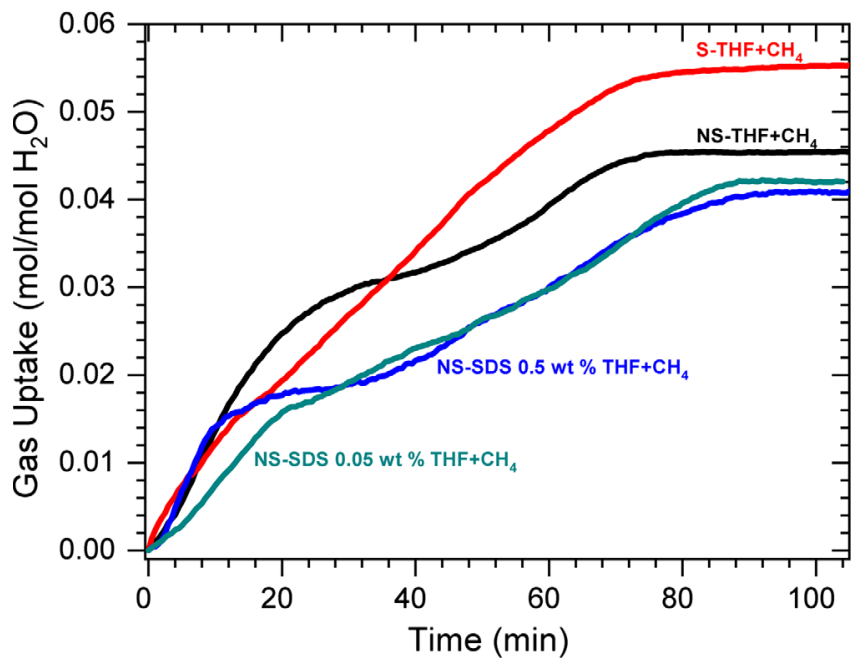

(b)

Fig. 6. a) Hydrate formation kinetics of mixed hydrates with $185 \mathrm{~mL}$ of aq-THF solution under Non-Stirred (NS) conditions at three different pressures. Standard deviation for three repeat measurements is shown as shaded portion. b) Formation kinetics of mixed hydrates under different experimental conditions. NS and $\mathrm{S}$ denote the non-stirred and stirred conditions. Also observed behaviour by adding of $0.5 \%$ and $0.05 \%$ SDS to aqTHF solution is shown in the graph.

in Figure 5 addition of SDS (green and blue bars) also is not helpful in increasing hydrate conversion. There is a marginal decrease in gas uptake. There are some conflicting reports on the role of SDS in the THF $+\mathrm{CH}_{4} / \mathrm{CO}_{2}$ system. Addition of SDS improves the kinetics in hydrate formation, while the gas consumption was reduced significantly [39]. On the other hand, simultaneous usage of SDS $(0.05 \mathrm{wt} \%)$ in aq-THF $(5 \mathrm{~mol} \%)$ in THF $+\mathrm{CO}_{2}$ hydrates allows faster nucleation and a significant increase in gas storage capacity [44]. Interestingly SDS also helps in promoting the methane gas uptake in hydrates formed with lesser $\mathrm{mol} \% \mathrm{THF}$, e.g., the gas uptake is $129.6 \mathrm{mmol} / \mathrm{mol}$, while there is no gas consumption in this system in the absence of SDS [26]. As noticed in the present study the gas uptake remained same in THF $+\mathrm{CH}_{4}$ system with or without SDS. Thus, more investigations are necessary for understanding the gas trapping mechanism in these hydrate forming systems.

\section{Kinetics of methane uptake at higher pressure}

Another important aspect is the kinetics of gas uptake in the hydrate process. In Figure 6a, we show the gas uptake ( $\mathrm{mol} / \mathrm{mol} \mathrm{H}_{2} \mathrm{O}$ ) with time in the reactor-2 at three pressures. Also, the kinetics for gas uptake is compared for $\mathrm{THF}+\mathrm{CH}_{4}$ in stirred and non-stirred conditions in Figure 6b. Observed kinetics for the THF $+\mathrm{CH}_{4}$ system along with 0.05 and 0.5 wt\% of SDS, in non-stirred configuration, is also included in Figure 6b. The time zero is from the onset of hydrate nucleation. It is worthwhile to note that there are no dramatic variations in the kinetics. The maximum gas consumption occurred within $60-70 \mathrm{~min}$ at all pressures under non-stirred conditions. The gas uptake under stirred conditions is marginally faster. Addition of SDS (0.05 and $0.5 \mathrm{wt} \%)$ induces insignificant variations in the kinetics. Interestingly, gas consumption is in two stages and similar behaviour has been reported earlier [38, 39].

\section{Conclusion}

Presently we demonstrate that the methane gas can be stored in mixed hydrates with THF rapidly in non-stirred configuration. We conducted experiments in two different capacity reactor vessels namely $100 \mathrm{~mL}(\phi=4.5 \mathrm{~cm})$ and $400 \mathrm{~mL}(\phi=7.5 \mathrm{~cm})$. Observed maximum methane gas consumption $(0.093$ and $0.417 \mathrm{~mol})$ occurred at half the reactor capacity. The effect of initial gas pressure on the methane gas uptake, in the range $0.5-10.3 \mathrm{MPa}$ range, is also investigated. The gas uptake increased by pressure; however, it is significantly less than the predicted gas uptake. There is an insignificant improvement in gas uptake in the experiments conducted under the stirred condition and also with SDS addition. The kinetics of the gas uptake process is also significantly faster (i.e., 60-70 min), paving the way for a larger number of hydrate formation cycles.

Acknowledgments. Authors sincerely thank the Director of the National Geophysical Research Institute, Hyderabad, for his encouragement, and permission to publish this paper (NGRI/ Lib/2018/113). Partial financial support from DST (India) and DGH-NGHP (India) is acknowledged.

\section{Supporting information}

The supporting information is available at http://ogst. ifpenergiesnouvelles.fr/10.2516/ogst/2018092/olm.

\section{References}

1 Sloan E.D., Koh C.A. (2008) Clatharate hydrates of natural gases, 3rd edn., CRC Press, Taylor \& Francis Group, Boca Raton. 
2 Thomas S., Dawe A.R. (2003) Review of ways to transport natural gas energy from countries which do not need the gas for domestic use, Energy 28, 1461-1477.

3 Englezos P., Lee J.D. (2005) Gas hydrates: A cleaner source of energy and opportunity for innovative technologies, Kor. J. Chem. Eng. 22, 671-681.

4 Gudmundsson J.S., Parlaktuna M., Khokhar A.A. (1994) Storage of natural gas as frozen hydrate, SPE Prod. Facil. 9, 69-73.

5 Prasad P.S.R., Eswari Ch.V.V. (2017) Chapter 11 Clathrate hydrates: A powerful tool to mitigate greenhouse gas, in: M. Goel, M. Sudhakar (eds), Carbon utilization Applications for the energy industry in India, Springer Nature, Singapore, pp. 157-168.

6 Zhao J., Zhao Y., Liang W. (2016) Hydrate-based gas separation for methane recovery from coal mine gas using tetrahydrofuran, Energy Technol. 4, 864-869.

7 Babu P., Linga P., Kumar R., Englezos P. (2015) A review of the hydrate based gas separation (HBGS) process for carbon dioxide pre-combustion capture, Energy 85, 261-279.

8 Bahadori A. (2014) Chapter 13 - Liquefied Natural Gas (LNG), in: A. Bahadori (ed), Natural Gas Processing, Gulf Professional Publishing, Boston, pp. 591-632.

9 Bekyarova E., Murata K., Yudasaka M., Kasuya D., Iijima S., Tanaka H., Kahoh H., Kaneko K. (2003) Single-wall nanostructured carbon for methane storage, J. Phys. Chem. $B$ 107, 4681-4684.

10 Zhou Y., He W., Qian G., Chen B. (2014) Methane storage in metal-organic frameworks, Chem. Soc. Rev. 43, 5657-5678.

11 Düren T., Sarkisov L., Yaghi O.M., Snurr R.Q. (2004) Design of new materials for methane storage, Langmuir $\mathbf{2 0}$, 2683-2689.

12 Eddaoudi M., Kim J., Rosi N., Vodak D., Wachter J., O'Keeffe M., Yaghi O.M. (2002) Systematic design of pore size and functionality in isoreticular MOFs and their application in methane storage, Science 295, 469-472.

13 Silvestre-Albero J., Wahby A., Sepulveda-Escribano A., Martinez-Escandell M., Kaneko K., Rodriguez-Reinoso F. (2011) Ultrahigh $\mathrm{CO}_{2}$ adsorption capacity on carbon molecular sieves at room temperature, Chem. Commun. 47, 6840-6842.

14 Prasad P.S.R., Chari V.D. (2015) Preservation of methane gas in the form of hydrates: Use of mixed hydrates, J. Nat. Gas Sci. Eng. 25, 10-14.

15 Ganji H., Manteghian M., Sadaghiani zadeh K., Omidkhaha M.R., Mofrad H.R. (2007) Effect of different surfactants on methane hydrate formation rate, stability and storage capacity, Fuel 86, 434-441.

16 Chari V.D., Sharma D.V.S.G.K., Prasad P.S.R. (2011) Methane hydrate phase stability with lower mole fractions of tetrahydro-furan (THF) and tert-butylamine $\left(t-\mathrm{BuNH}_{2}\right)$, Fluid Phase Equilib. 315, 126-130.

17 Kang S.P., Lee J.W. (2010) Formation characteristics of synthesized natural gas hydrates in meso- and macroporous silica gels, J. Phys. Chem. B 114, 6973-6978.

18 Chari V.D., Raju B., Prasad P.S.R., Rao D.N. (2013) Methane hydrates in spherical silica matrix: Optimization of capillary water, Energy Fuels 27, 3679-3684.

19 Chari V.D., Sharma D.V.S.G.K., Prasad P.S.R., Murthy S.R. (2013) Methane hydrates formation and dissociation in nano silica suspension, J. Nat. Gas Sci. Eng. 11, 7-11.
20 Sowjanya K., Prasad P.S.R. (2016) Sustainability of hollow silica matrix for clathrate hydrate recycling, J. Nat. Gas Sci. Eng. 34, 585-589.

21 Chari V.D., Prasad P.S.R., Murthy S.R. (2014) Structural stability of methane hydrates in porous medium: Raman spectroscopic study, Spectrochim. Acta A. 120, 636-641.

22 Prasad P.S.R., Sowjanya Y., Chari V.D. (2014) Enhancement in methane storage capacity in gas hydrates formed in hollow silica, Phys. Chem. C 118, 7759-7764.

23 Florusse L.J., Peters C.J., Schoonman J., Hester K.C., Koh C.A., Dec S.F., Marsh K.N., Sloan E.D. (2004) Stable lowpressure hydrogen clusters stored in a binary clathrate hydrate, Science 306, 469-471.

24 Strobel T.A., Koh C.A., Sloan E.D. (2009) Thermodynamic predictions of various tetrahydrofuran and hydrogen clathrate hydrates, Fluid Phase Equilib. 280, 61-67.

25 Delahaye A., Fournaison L., Marinhas S., Chatti I., Petitet J.P., Dalmazzone D., Fürst W. (2006) Effect of THF on equilibrium pressure and dissociation enthalpy of $\mathrm{CO}_{2}$ hydrates applied to secondary refrigeration, Ind. Eng. Chem. Res. 45, 391-397.

26 Siangasi A., Inkong K., Kulprathipanja S., Kitiyanan B., Rangsunvigit P. (2018) Role of sodium dodecyl sulfate on tetrahydrofuran-assisted methane hydrate formation, J. Olea Sci. 67, 707-717.

27 Ricaurte M., Dicharry C., Renaud X., Torre J.P. (2014) Combination of surfacants and organic compounds for boosting $\mathrm{CO}_{2}$ separation from natural gas by clathrate hydrate formation, Fuel 122, 206-217.

28 Ricaurte M., Torre J.P., Diaz J., Dicharry C. (2014) In situ injection of $\mathrm{THF}$ to trigger gas hydrate crystallization: Application to the evaluation of a kinetic hydrate promoter, Chem. Eng. Res. Des. 92, 1674-1680.

29 Dicharry C., Diaz J., Torre J.P., Ricaurte M. (2016) Influence of the carbon chain length of a sulfate-based surfactant on the formation of $\mathrm{CO}_{2}, \mathrm{CH}_{4}$ and $\mathrm{CO}_{2}-\mathrm{CH}_{4}$ gas hydrates, Chem. Eng. Sci. 152, 736-745.

30 Lee Y.J., Kawamura T., Yamamoto Y., Yoon J.H. (2012) Phase equilibrium studies of tetrahydrofuran (THF) $+\mathrm{CH}_{4}$, $\mathrm{THF}+\mathrm{CO}_{2}, \mathrm{CH}_{4}+\mathrm{CO}_{2}$, and THF $+\mathrm{CO}_{2}+\mathrm{CH}_{4}$ Hydrates, J. Chem. Eng. Data 57, 3543-3548.

31 Seo Y.T., Lee H., Moudrakovaski I., Ripmeester J.A. (2003) Phase behavior and structural characterization of coexisting pure and mixed clathrate hydrates, Chem. Phys. Chem. 4, 379-382.

32 Mohammadi A.H., Richon D. (2009) Phase equilibria of clathrate hydrates of tetrahydrofuran + hydrogen sulfide and tetrahydrofuran + methane, Ind. Eng. Chem. Res. 48, 7838-7841.

33 Zhang Q., Chen G.J., Huang Q., Sun C.Y., Guo X.Q., Ma Q.L. (2005) Hydrate formation conditions of a hydrogen + methane gas mixture in tetrahydrofuran + water, J. Chem. Eng. Data 50, 234-236.

34 De Deugd R., Jager M., de Arons S.J., (2001) Mixed hydrates of methane and water-soluble hydrocarbons modeling of empirical results, AIChE J. 47, 693-704.

35 Seo Y.T., Kang S.P., Lee H. (2001) Experimental determination and thermodynamic modeling of methane and nitrogen hydrates in the presence of THF, propylene oxide, 1,4-dioxane and acetone, Fluid Phase Equilib. 189, 99-110.

36 Luo Y.T., Zhu J.H., Fan S.S., Chen G.J. (2007) Study on the kinetics of hydrate formation in a bubble column, Chem. Eng. Sci. 62, 1000-1009. 
37 Sharma D., Sowjanya Y., Chari V.D., Prasad P.S.R. (2014) Methane storage in mixed hydrates with tetrahydrofuran, Indian J. Chem. Technol. 21, 114-119.

38 Veluswamy H.P., Wong A.J.H., Babu P., Kumar R., Kulprathipanja S., Rangsunvigit P., Linga P. (2016) Rapid methane hydrate formation to develop a cost effective large scale energy storage system, Chem. Eng. J. 290, 161-173.

39 Veluswamy H.P., Kumar S., Kumar R., Rangsunvigit P., Linga P. (2016) Enhanced clathrate hydrate formation kinetics at near ambient temperatures and moderate pressures: Application to natural gas storage, Fuel 182, 907-919.

40 Larionov E.G., Manakov A.Y., Zhurko F.V., Dyadin Y.A. (2000) Double clathrate hydrate CS-II at pressures up to 15 kbar, J. Struct. Chem. 41, 476-482.
$41 \mathrm{Ke}$ W., Svartaas T.M. (2011) The effect of molar liquid water-gas ratio on methane hydrate formation, Proc. 7th Int. Conf. Gas Hydrates, ICGH-7, Edinburgh, UK, July $17-21$.

42 Jung J.-W., Carlos S.J. (2012) Hydrate formation and growth in pores, J. Cryst. Growth 345, 61-68.

43 Yoon J.-H. (2012) A theoretical prediction of cage occupancy and heat of dissociation of $\mathrm{THF}-\mathrm{CH}_{4}$ hydrate, Kor. J. Chem. Eng. 29, 1670-1673.

44 Lirio C.F.S., Pessoa F.L.P., Uller A.M.C. (2013) Storage capacity of carbon dioxide hydrates in the presence of sodium dodecyl sulfate (SDS) and tetrahydrofuran (THF), Chem. Eng. Sci. 96, 118-123. 